\title{
Parâmetros nutricionais e padrões nictemeral do comportamento ingestivo de cordeiros Somalis Brasileira alimentados com diferentes níveis de energia metabolizável
}

\author{
[Nutritional parameters and nycterohemeral patterns of ingestive behavior of Brazilian \\ Somali lambs fed different levels of metabolizable energy] \\ R.M.S. Aquino ${ }^{1}$, E.S. Pereira ${ }^{2 *}$, S.M.P. Oliveira $^{2}$, P.G. Pimentel ${ }^{2}$, I.Y. Mizubuti ${ }^{3}$, E.L.A, Ribeiro ${ }^{3}$, \\ M.S.S. Carneiro ${ }^{2}$, M.J.D. Cândido ${ }^{2}$, R.M. Fontenele ${ }^{1}$, J.P.M. Alves ${ }^{4}$, \\ R.O. Cruz ${ }^{4}$ J.N. Rocha Júnior ${ }^{2}$ \\ ${ }^{1}$ Aluna de pós-graduação - Universidade Federal do Ceará - Fortaleza, CE \\ ${ }^{2}$ Universidade Federal do Ceará - Fortaleza, CE \\ ${ }^{3}$ Universidade Estadual de Londrina - Londrina, PR \\ ${ }^{4}$ Aluna de Iniciação Científica - Universidade Federal do Ceará - Fortaleza, CE
}

\begin{abstract}
RESUMO
O presente estudo foi conduzido para determinar o consumo e a digestibilidade dos nutrientes, bem como o comportamento ingestivo de cordeiros Somalis Brasileira, alimentados com diferentes níveis de energia metabolizável (EM). Foram utilizados 40 animais, não castrados, com peso vivo médio de 13,47士1,76kg. Os animais foram distribuídos em delineamento em blocos ao acaso, com cinco dietas com diferentes níveis de energia metabolizável $(1,18 ; 2,07 ; 2,25 ; 2,42$ e 2,69Mcal $/ \mathrm{kg}$ de MS). O consumo de MS, PB, FDN, CF (g/dia), MS ( $\left./ \mathrm{kg}^{0,75}\right)$ e FDN (\%PC e g/kg $\left.{ }^{0,75}\right)$ apresentaram efeito quadrático $(\mathrm{P}<0,05)$, assim como os coeficientes de digestibilidade $(\mathrm{P}<0,05)$ da $\mathrm{PB}$, da FDN e dos CF. Foi averiguado efeito linear crescente $(\mathrm{P}<0,05)$ para digestibilidade da MS, da MO e dos CNF com o aumento da EM na dieta. Os tempos de alimentação e ócio (h/dia) foram influenciados pelos níveis de energia $(\mathrm{P}<0,05)$, e as eficiências de alimentação e ruminação ( $\mathrm{g} F \mathrm{FN} / \mathrm{h})$ apresentaram efeito quadrático $(\mathrm{P}<0,05)$. Conclui-se que o aumento dos níveis de EM das rações influencia o consumo e a digestibilidade dos nutrientes, bem como o comportamento ingestivo de cordeiros Somalis Brasileira.
\end{abstract}

Palavras-chave: consumo, cordeiros, nível de alimentação, tempo de alimentação, tempo de ruminação

\begin{abstract}
The study was conducted to determine the intake, nutrient digestibility and ingestive behavior of Brazilian Somali lambs fed different levels of metabolizable energy (ME). Forty non-castrated Brazilian Somali lambs with average weight of $13.47 \pm 1.76 \mathrm{~kg}$ were used. The animals were assigned to a randomized block design with eight replications per block and five diets with increasing metabolizable energy (1.18, 2.07, 2.25, 2.42 and $2.69 \mathrm{Mcal} / \mathrm{kg} D \mathrm{DM})$. The quadratic effect $(P<0.05)$ of the ME level was determined for DM, $C P, N D F$ and $F C\left(g /\right.$ day) and $D M\left(\mathrm{~g} \mathrm{~kg}^{0,75}\right)$ and $\mathrm{NDF}\left(\% \mathrm{PC}\right.$ and $\left.\mathrm{g} / \mathrm{kg}^{0,75}\right)$ intake, as well as the digestibility coefficients $(P<0.05)$ for $C P, N D F$ and $C F$. A linear effect $(P<0.05)$ was observed for digestibility of DM, OM and NFC with the increase of ME in the diet. Eating and idle results (hours/day) were influenced by the energy levels $(P<0.05)$ and efficiencies of feeding and rumination $(g N D F / h)$ showed a quadratic effect $(P<0.05)$. It is concluded that the increase of ME levels in the diet influences intatke, nutrient digestibility and ingestive behavior of Brazilian Somali lambs.
\end{abstract}

Keywords: intake, lambs, feeding level, eating time, rumination time

Recebido em 30 de abril de 2012

Aceito em 11 de novembro de 2013

*Autor para correspondência (corresponding author)

E-mail: elzania@hotmail.com 


\section{INTRODUÇÃO}

Entre os fatores que influenciam a eficiência produtiva em sistemas de produção de ruminantes, o custo de alimentação dos animais é a mais importante variável dos custos operacionais de produção. Desta maneira, o conhecimento do consumo de matéria seca das rações possibilita maior previsibilidade das respostas produtivas dos animais. A partir do conhecimento quantitativo dos nutrientes, poderão ser balanceadas dietas que resultem em maior eficiência dos sistemas de produção, por minimizar os custos com a alimentação e as perdas em nutrientes (Russell et al., 1992; Sniffen et al., 1992).

Vários fatores influenciam o consumo voluntário de nutrientes. Teorias individuais baseadas em enchimento físico do rúmen, fatores relacionados a feedback metabólico ou consumo de oxigênio, têm sido utilizadas para determinação e/ou predição do consumo de matéria seca (Nutrient..., 2007). Segundo Mertens (1994), o desempenho animal é função direta do consumo de matéria seca digestível. Nessa circunstância, 60 a $90 \%$ do desempenho decorrem da variação do consumo, enquanto 10 a $40 \%$ provêm de flutuações na digestibilidade. Uma vez que as características de digestibilidade são intrínsecas ao alimento, bem como a sua composição, o consumo e sua intensidade assumem particular importância nos sistemas de produção animal. Sendo assim, o consumo é considerado o fator mais importante na determinação do desempenho animal. Em tal contexto, o estudo do comportamento ingestivo pode propiciar nova perspectiva para o modelo convencional de abordagem científica zootécnica, abrindo novos horizontes e trazendo inovações a situações não consideradas ou mal compreendidas, principalmente no que se refere às práticas de manejo (Silva et al., 2004).

O objetivo deste trabalho foi, portanto, avaliar o consumo e a digestibilidade dos nutrientes e sua relação com as variáveis de comportamento ingestivo de cordeiros da raça Somalis Brasileira em confinamento, alimentados com dietas com diferentes níveis de energia.

\section{MATERIAL E MÉTODOS}

O experimento foi desenvolvido no Setor de Digestibilidade do Departamento de Zootecnia da Universidade Federal do Ceará, Fortaleza-CE. Foram utilizados 40 cordeiros da raça Somalis Brasileira, não castrados, com peso vivo médio inicial de $13,47 \mathrm{~kg} \pm 1,76 \mathrm{~kg}$ e, aproximadamente, 60 dias de idade. Inicialmente, os animais foram pesados, identificados e vermifugados. Posteriormente, foram alocados em baias individuais contendo comedouros e bebedouros. O período experimental teve duração de 81 dias, sendo 14 dias de adaptação dos animais às dietas experimentais e às baias.

Os cordeiros $(n=40)$ foram alocados em cinco tratamentos experimentais, que consistiram em diferentes níveis de energia metabolizável $(1,18$; 2,07; 2,25; 2,42 e 2,69 Mcal/kg de MS) obtidos a partir de diferentes relações volumoso: concentrado (100:00; 80:20; 60:40; 40:60 e 20:80).

As rações experimentais foram formuladas conforme o Nutrient... (2007) para conterem em média $16 \%$ de proteína bruta e promover ganho de $200 \mathrm{~g} / \mathrm{dia}$, exceto para a relação 100:0, sendo esta formulada para atender a exigência de mantença com 9\% de proteína bruta. A composição química dos ingredientes e a composição percentual e química das rações experimentais estão apresentadas nas Tab. 1 e 2, respectivamente.

O fornecimento das rações foi realizado duas vezes ao dia, às oito horas e às $16 \mathrm{~h}$. A quantidade de alimento oferecido e das sobras foi registrada diariamente, a fim de se determinar o consumo de alimento, e realizou-se o ajuste para permitir sobras em torno de $10 \%$ do fornecido. A água foi colocada permanentemente à disposição dos animais. Semanalmente, foram coletadas amostras do feno, concentrados e sobras da ração total, sendo estas acondicionadas em sacos plásticos, devidamente identificadas e armazenadas em freezer $\left(-10^{\circ} \mathrm{C}\right)$, onde, ao final do período experimental, formaram uma amostra composta/tratamento/animal para posteriores análises. 
Parâmetros nutricionais...

Tabela 1. Composição química dos ingredientes em \% MS

\begin{tabular}{lccccccc} 
Nutriente $^{1}$ & Feno de & Milho Grão & Farelo de & \multicolumn{5}{c}{ Concentrados } \\
\cline { 7 - 8 } & Tifton 85 & moído & soja & 1 & 2 & 3 & 4 \\
\hline MS & 92,02 & 92,44 & 91,79 & 91,92 & 92,12 & 91,98 & 91,93 \\
MO & 94,37 & 97,98 & 93,29 & 94,07 & 96,74 & 96,71 & 96,38 \\
PB & 9,25 & 7,99 & 42,74 & 45,08 & 26,21 & 21,47 & 18,88 \\
EE & 2,33 & 6,48 & 2,22 & 2,99 & 5,00 & 5,99 & 5,60 \\
MM & 5,63 & 2,02 & 6,71 & 5,93 & 3,26 & 3,29 & 3,62 \\
FDN & 79,10 & 11,42 & 19,00 & 16,94 & 13,16 & 12,15 & 12,10 \\
FDA & 35,24 & 3,37 & 10,49 & 8,54 & 5,76 & 5,49 & 5,45 \\
Lignina & 4,49 & 0,75 & 0,86 & 0,96 & 0,37 & 1,47 & 0,71 \\
Celulose & 31,38 & 2,78 & 9,85 & 7,92 & 5,83 & 4,50 & 5,64 \\
Hemicelulose & 43,86 & 8,05 & 8,51 & 8,40 & 7,40 & 6,65 & 6,65 \\
CT & 82,79 & 83,51 & 48,33 & 46,00 & 59,57 & 69,24 & 70,53 \\
CF & 72,18 & 9,42 & 11,96 & 11,81 & 10,09 & 9,11 & 8,50 \\
CNF & 10,61 & 74,09 & 36,37 & 39,62 & 51,75 & 62,16 & 62,95 \\
\hline
\end{tabular}

Tabela 2. Composição percentual e química das rações experimentais

\begin{tabular}{lccccc}
\hline \multirow{2}{*}{ Ingrediente (\%MN) } & \multicolumn{5}{c}{ Níveis de EM (Mcal/kg MS) } \\
\cline { 2 - 5 } & 1,18 & 2,07 & 2,25 & 2,42 & 2,69 \\
\hline Feno de Tifton & 100 & 80 & 60 & 40 & 20 \\
Concentrado & 0 & 20 & 40 & 60 & 80 \\
Milho grão moído & - & 15,87 & 69,45 & 72,46 & 75,61 \\
Farelo de soja $^{1}$ & - & 80,65 & 28,53 & 24,88 & 22,59 \\
Uréia $^{1}$ & - & 3,00 & 1,25 & 1,12 & 0,50 \\
Calcário $^{1}$ & - & - & - & 0,54 & 0,66 \\
Fosfato bicálcico $^{1}$ & - & - & - & - & 0,07 \\
Cloreto de sódio & & 0,40 & 0,70 & 0,93 & 0,50 \\
Mistura Mineral ${ }^{1,2}$ & - & 0,08 & 0,07 & 0,07 & 0,06 \\
\hline & - & 92,00 & 92,06 & 92,00 & 91,95 \\
\hline MS & 92,02 & 94,31 & 95,32 & 95,77 & 95,98 \\
MO & 94,37 & 5,69 & 4,68 & 4,23 & 4,02 \\
MM & 5,63 & 16,42 & 16,03 & 16,58 & 16,95 \\
PB & 9,25 & 2,46 & 3,40 & 4,53 & 4,95 \\
EE & 2,33 & 66,67 & 52,72 & 38,93 & 25,50 \\
FDN & 79,10 & 29,90 & 23,45 & 17,39 & 11,40 \\
FDA & 35,24 & 3,78 & 2,84 & 2,67 & 1,46 \\
Lignina & 4,49 & 26,69 & 21,16 & 15,25 & 10,79 \\
Celulose & 31,38 & 36,77 & 29,27 & 21,54 & 14,09 \\
Hemicelulose & 43,86 & 75,43 & 75,89 & 74,66 & 74,08 \\
CT & 82,79 & 60,11 & 47,35 & 34,34 & 21,24 \\
CF & 72,18 & 16,41 & 27,06 & 41,54 & 52,48 \\
CNF & 10,61 & 57,68 & 60,88 & 66,85 & 74,50 \\
NDT & 34,78 & 3,51 & 3,80 & 4,03 & 4,39 \\
NDT:PB & 3,76 & & &
\end{tabular}

${ }^{1}$ Composição centesimal em relação à porção concentrada da dieta.

${ }^{2}$ Composição: Ca 7,5\%; P 3\%; Fe 16.500mg/kg, Mn 9.750mg/kg, Zn 35.000mg/kg, I 1.000mg/kg, Se 225mg/kg, Co $1.000 \mathrm{mg} / \mathrm{kg}$.

Para determinação da digestibilidade dos nutrientes, a fibra em detergente neutro indigestível (FDNi) foi utilizada como indicador para estimar a produção de matéria seca fecal, conforme descrito por Casali et al. (2009). As fezes foram coletadas diretamente na ampola retal dos animais a cada 15 dias, durante três dias consecutivos: às oito horas no primeiro dia, às $12 \mathrm{~h}$ no segundo dia e às $17 \mathrm{~h}$ no terceiro dia, obtendo-se 12 amostras por animal. Ao final do 
período experimental, formaram-se amostras compostas por tratamento/animal. Amostras de fezes e alimentos foram pré-secas a $55^{\circ} \mathrm{C}$, moídas a $2 \mathrm{~mm}$ e, proporcionalmente, subamostradas, formando uma amostra composta por animal/tratamento, incubadas no rúmen, em sacos de náilon por um período de $240 \mathrm{~h}$, e posteriormente analisadas.

As amostras de feno, ingredientes, concentrados, sobras e fezes foram pré-secas em estufa ventilada a $55^{\circ} \mathrm{C}$ e moídas em moinho tipo Willey, com peneira de malha de $1 \mathrm{~mm}$, sendo estas submetidas às análises de matéria seca (MS), matéria mineral (MM), proteína bruta (PB) e extrato etéreo (EE), conforme os procedimentos recomendados pela AOAC (Official...1990), e fibra em detergente ácido (FDA), conforme Van Soest et al. (1991). Para análise de fibra em detergente neutro (FDN), as amostras foram tratadas com alfa-amilase, sendo corrigida para cinzas residuais e para compostos nitrogenados residuais. Os teores de carboidratos totais, com base na matéria seca, foram calculados pela fórmula: CT $(\%=100-(\% \mathrm{~PB}+$ $\% \mathrm{EE}+\%$ cinzas), segundo Sniffen et al. (1992), e os carboidratos não fibrosos foram calculados conforme equação proposta por Weiss (1999): $\mathrm{CNF}(\%)=100-(\% \mathrm{FDNcp}+\% \mathrm{~PB}+\% \mathrm{EE}+$ \%cinzas). Para os concentrados, devido à presença de ureia em sua constituição, o teor de CNF foi calculado conforme proposto por Hall (2000), sendo $\mathrm{CNF}=100-[(\% \mathrm{~PB}-\% \mathrm{~PB}$ derivado da ureia $+\%$ da ureia) $+\%$ FDNcp + $\% \mathrm{EE}+\%$ cinzas]. $\mathrm{O}$ teor de nutrientes digestíveis totais foi calculado de acordo com Weiss (1999): $\mathrm{NDT}=\mathrm{PBd}+\mathrm{CNFd}+\mathrm{FDNcpd}+\mathrm{EEd} \times 2,25$, em que PBd, CNFd, FNDcpd e EEd correspondem a proteína bruta digestível, carboidratos não fibrosos digestíveis, fibra em detergente neutro corrigida para cinzas e proteína digestível e extrato etéreo digestível, respectivamente.

A energia digestível (ED) da dieta foi estimada em 4,409kg/Mcal de NDT e foi convertida para energia metabolizável (EM) usando-se uma eficiência de $82 \%$ (Nutrient..., 2000).

Para mensuração do comportamento ingestivo, os animais foram submetidos à observação visual no $73^{\circ}$ e no $74^{\circ}$ dia. No primeiro dia, foi realizada observação visual, em intervalos de cinco minutos durante 24 horas, para determinação do tempo despendido com alimentação, ruminação e ócio, conforme metodologia proposta por Johnson e Combs (1991). O galpão foi mantido sob iluminação artificial à noite durante todo $o$ período experimental. No segundo dia, os animais foram observados durante três períodos de duas horas (das oito às 10h; 14 às $16 \mathrm{~h}$ e 18 às 20h), sendo coletadas informações para se estimar o número de mastigações merícicas por bolo ruminal e o tempo despendido com mastigação merícica por bolo ruminal, utilizando-se cronômetro digital. As variáveis do comportamento ingestivo foram obtidas pelas equações: $\mathrm{EAL}=\mathrm{CMS} / \mathrm{TAL}$; $\mathrm{EAL}=\mathrm{CFDN} / \mathrm{TAL} ; \mathrm{ERU}=\mathrm{CMS} / \mathrm{TRU}$; $\mathrm{ERU}=\mathrm{CFDN} / \mathrm{TRU} ; \mathrm{TMT}=\mathrm{TAL}+\mathrm{TRU} ;$ NBR = TRU/MMtb; MMnd = NBR $*$ MMnb, em que: EAL (g MS/h, g FDN/h) corresponde à eficiência de alimentação; CMS (g MS/dia) corresponde ao consumo de MS; TAL (h/dia) corresponde ao tempo de alimentação; CFDN (g FDN/dia) corresponde ao consumo de FDN; ERU (g MS/h, g FDN/h) corresponde à eficiência de ruminação; TRU (h/dia) corresponde ao tempo de ruminação; TMT (h/dia) corresponde ao tempo de mastigação total; NBR ( $\left.\mathrm{n}^{\circ} / \mathrm{dia}\right)$ corresponde ao número de bolos ruminais; MMtb (seg/bolo) corresponde ao tempo de mastigação merícica por bolo ruminal, MMnb ( $\left.\mathrm{n}^{\circ} / \mathrm{bolo}\right)$ corresponde ao número de mastigações merícicas por bolo ruminal; e MMnd ( $\mathrm{n}^{\circ} /$ bolo) corresponde ao número de mastigações por dia (Polli et al., 1996).

O delineamento experimental foi em blocos ao acaso (peso corporal inicial), com cinco tratamentos, conforme descrito anteriormente, de acordo com o modelo matemático Yij $=\mu+\alpha \mathrm{i}+$ $\beta \mathrm{j}+$ eij, em que Yij = valor observado na parcela que recebeu o tratamento i no bloco $\mathrm{j}=1,2,3,4$, 5, 6, 7, 8; eij = erro aleatório.

As análises estatísticas foram realizadas utilizando-se o PROC GLM SAS, versão 9.0 (Statistical, 2003).

\section{RESULTADOS E DISCUSSÃO}

Foi observado efeito dos níveis de energia metabolizável (EM; $\mathrm{P}<0,05)$ sobre o consumo de matéria seca, expresso em g/dia e $\mathrm{g} / \mathrm{kg}^{0,75}$, apresentando efeito quadrático (Tab. 3). Os consumos máximos de MS estimados foram 
$684,64 \mathrm{~g} / \mathrm{dia}$ e $70,58 \mathrm{~g} / \mathrm{kg}^{0,75}$, para os níveis de 2,69 e 2,42Mcal $/ \mathrm{kg}$ de MS, respectivamente. Entretanto, foi observado efeito linear crescente $(\mathrm{P}<0,05)$ para o consumo de $\mathrm{MS}$, expresso em $\% \mathrm{PC}$ (Tab. 3). No que se refere ao consumo de
MO, EE, CNF e NDT, expressos em g/dia, foi observado efeito linear crescente $(\mathrm{P}<0,001$; Tab. 3), o que pode ser explicado pelo fato de haver maior concentração destes nutrientes nas dietas (Tab. 2).

Tabela 3. Consumo de nutrientes em cordeiros Somalis Brasileira alimentados com rações com diferentes níveis de energia metabolizável

\begin{tabular}{|c|c|c|c|c|c|c|c|c|c|}
\hline \multirow{2}{*}{$\begin{array}{l}\text { Variáve } \\
\text { is }\end{array}$} & \multicolumn{5}{|c|}{ Níveis de EM (Mcal/kg de MS) } & \multirow{2}{*}{ EPM } & \multicolumn{3}{|c|}{ Efeito } \\
\hline & 1,18 & 2,07 & 2,25 & 2,42 & 2,69 & & $\mathrm{~L}$ & $\mathrm{Q}$ & $\mathrm{C}$ \\
\hline \multicolumn{10}{|c|}{ Consumo (g/dia) } \\
\hline $\mathrm{MS}^{1}$ & 390,52 & 549,45 & 695,00 & 762,15 & 636,83 & 22,18 & $<0,0001$ & 0,0334 & 0,0005 \\
\hline $\mathrm{MO}^{2}$ & 332,16 & 517,32 & 664,04 & 732,26 & 631,65 & 23,64 & $<0,0001$ & 0,0446 & 0,6578 \\
\hline $\mathrm{PB}^{3}$ & 32,43 & 104,75 & 134,93 & 135,09 & 127,18 & 6,55 & $<0,0001$ & 0,0106 & 0,1725 \\
\hline $\mathrm{EE}^{4}$ & 8,40 & 14,76 & 24,83 & 35,16 & 32,36 & 1,67 & $<0,0001$ & 0,0378 & 0,0589 \\
\hline $\mathrm{FDN}^{5}$ & 321,35 & 377,12 & 341,61 & 269,96 & 153,84 & 14,75 & 0,0017 & $<0,0001$ & 0,8598 \\
\hline $\mathrm{CT}^{6}$ & 287,65 & 401,03 & 495,07 & 538,29 & 462,74 & 15,96 & $<0,0001$ & 0,0698 & $<0,0001$ \\
\hline $\mathrm{CF}^{7}$ & 254,85 & 338,08 & 305,37 & 239,3 & 127,68 & 13,37 & 0,0194 & $<0,0001$ & 0,8299 \\
\hline $\mathrm{CNF}^{8}$ & 36,82 & 100,50 & 207,55 & 342,94 & 375,06 & 21,48 & $<0,0001$ & $<0,0001$ & $<0,0001$ \\
\hline $\mathrm{NDT}^{9}$ & 144,47 & 318,55 & 434,70 & 511,20 & 481,09 & 21,56 & $<0,0001$ & 0,6561 & $<0,0001$ \\
\hline \multicolumn{10}{|c|}{ Consumo (\%PC) } \\
\hline $\mathrm{MS}^{10}$ & 2,68 & 3,04 & 3,64 & 3,59 & 3,38 & 0,07 & $<0,0001$ & 0,1475 & 0,0004 \\
\hline $\mathrm{FDN}^{11}$ & 2,27 & 2,17 & 1,82 & 1,30 & 0,78 & 0,10 & $<0,0001$ & $<0,0001$ & 0,0595 \\
\hline \multicolumn{10}{|c|}{ Consumo $\left(\mathrm{g} / \mathrm{kg}^{0,75}\right)$} \\
\hline $\mathrm{MS}^{12}$ & 52,12 & 62,70 & 75,75 & 76,94 & 67,13 & 1,63 & $<0,0001$ & 0,0115 & 0,0809 \\
\hline $\mathrm{FDN}^{13}$ & 43,91 & 44,24 & 37,84 & 27,73 & 16,49 & 1,85 & $<0,0001$ & $<0,0001$ & 0,1166 \\
\hline
\end{tabular}

EPM = erro padrão da média; $\mathrm{L}=$ efeito linear; $\mathrm{Q}=$ efeito quadrático; $\mathrm{C}=$ efeito cúbico.

${ }^{1} \hat{Y}=-277,72+714,97 \mathrm{EM}-132,78 \mathrm{EM}^{2}\left(\mathrm{R}^{2}=0.64\right) ;{ }^{2} \hat{\mathrm{Y}}=59,84+243,17 \mathrm{EM}\left(\mathrm{R}^{2}=0.69\right) ;{ }^{3} \hat{\mathrm{Y}}=-197,57+249,87 \mathrm{EM}^{2}-47,38 \mathrm{EM}^{2}$ $\left(\mathrm{R}^{2}=0.82\right) ; \quad{ }^{4} \hat{\mathrm{Y}}=11,42-12,60 \mathrm{EM}+8,06 \mathrm{EM}^{2} \quad\left(\mathrm{R}^{2}=0.76\right) ; \quad{ }^{5} \hat{\mathrm{Y}}=-447,93+987,38 \mathrm{EM}-284,18 \mathrm{EM}^{2} \quad\left(\mathrm{R}^{2}=0.74\right) ;$ ${ }^{6} \hat{\mathrm{Y}}=125,73+146,80 \mathrm{EM} \quad\left(\mathrm{R}^{2}=0.55\right) ; \quad{ }^{7} \hat{\mathrm{Y}}=-563,99+1036,09 \mathrm{EM}-289,84 \mathrm{EM}^{2} \quad\left(\mathrm{R}^{2}=0.77\right) ; \quad{ }^{8} \hat{\mathrm{Y}}=-283,15+233,08 \mathrm{EM}$ $\left(\mathrm{R}^{2}=0.76\right) ; \quad{ }^{9} \hat{\mathrm{Y}}=-139,05+244,32 \mathrm{EM} \quad\left(\mathrm{R}^{2}=0.83\right) ; \quad{ }^{10} \hat{\mathrm{Y}}=2,03+0,58 \mathrm{EM} \quad\left(\mathrm{R}^{2}=0.68\right) ; \quad{ }^{11} \hat{\mathrm{Y}}=-0,76+4,15 \mathrm{EM}-1,34 \mathrm{EM}^{2}$ $\left(\mathrm{R}^{2}=0.85\right) ;{ }^{12} \hat{\mathrm{Y}}=-5,12+63,30 \mathrm{EM}-13,07 \mathrm{EM}^{2}\left(\mathrm{R}^{2}=0.55\right) ;{ }^{13} \hat{\mathrm{Y}}=-25,19+92,78 \mathrm{EM}-28,88 \mathrm{EM}^{2}\left(\mathrm{R}^{2}=0.85\right)$.

Resultados semelhantes foram obtidos por Fontenele et al. (2011), ao estudarem rações com diferentes níveis de energia metabolizável $(2,08$; 2,$28 ; 2,47$ e 2,69Mcal de EM/kg de MS) para cordeiros da raça Santa Inês. O consumo de PB, FDN e CF, expressos em g/dia, foi influenciado de forma quadrática pelos níveis de energia metabolizável na dieta, em que foram estimados os pontos máximos de consumo, correspondentes aos seguintes níveis de energia: 2,64, 1,74 e $1,79 \mathrm{Mcal} / \mathrm{kg}$ de MS, respectivamente (Tab. 3). Camilo et al. (2012), ao trabalharem com níveis crescentes de energia metabolizável $(0,96 ; 1,28$; 1,$72 ; 2,18$ e $2,62 \mathrm{Mcal} / \mathrm{kg}$ de MS) em cordeiros Morada Nova, registraram efeito quadrático para o consumo de FDN e CT, expressos em g/dia. O consumo de FDN, em \%PC e kg ${ }^{0,75}$, apresentou efeito quadrático $(\mathrm{P}<0,001)$ com pontos de máxima de 1,55 e $1,61 \mathrm{Mcal} / \mathrm{kg}$ de $\mathrm{MS}$, respectivamente. A relação entre a ingestão de MS e o conteúdo de FDN na ração é quadrática, o que mostra existir um ponto de inflexão entre o controle físico e o fisiológico, no qual o efeito da FDN sobre a ingestão cessa, e esta passa a depender do requerimento do animal. Segundo Mertens (1994), a base para expressar consumo não é semelhante para os mecanismos físicos e fisiológicos de controle. O ideal é expressar em \%PC quando se refere a rações de baixa qualidade, uma vez que a ingestão é limitada pelo enchimento do rúmen e está mais relacionada ao tamanho e à capacidade do trato digestório. Porém, quando o consumo é limitado pela demanda fisiológica de energia, a melhor forma de expressá-lo é com base no peso metabólico. O mecanismo de regulação fisiológica pode ser interpretado em uma situação em que, no consumo de matéria seca, a concentração energética da dieta seja igual ao requerimento animal (Mertens, 1994).

Os coeficientes de digestibilidade aparente de MS, MO, CT e CNF foram influenciados de forma linear crescente $(\mathrm{P}<0,001)$ com $\mathrm{O}$ 
incremento de energia metabolizável na dieta (Tab. 4). De acordo com Valadares Filho (1985), a digestibilidade aparente dos carboidratos não estruturais é acima de $90 \%$ e a dos carboidratos estruturais é próxima de $50 \%$. Porém, o excesso de CNF merece atenção especial, pois, por serem estes prontamente fermentáveis, podem comprometer a atividade microbiana no rúmen quando em altas quantidades, principalmente no que se refere à atividade das bactérias fibrolíticas, prejudicando, assim, o processo de digestão.

Tabela 4. Digestibilidade de nutrientes em cordeiros Somalis Brasileira alimentados com rações com diferentes níveis de energia metabolizável

\begin{tabular}{|c|c|c|c|c|c|c|c|c|c|}
\hline \multirow{2}{*}{$\begin{array}{c}\text { Variáveis } \\
(\%)\end{array}$} & \multicolumn{5}{|c|}{ Níveis de EM (Mcal/kg de MS) } & \multirow{2}{*}{ EPM } & \multicolumn{3}{|c|}{ Efeito } \\
\hline & 1,18 & 2,07 & 2,25 & 2,42 & 2,69 & & $\mathrm{~L}$ & $\mathrm{Q}$ & $\mathrm{C}$ \\
\hline $\mathrm{MS}^{1}$ & 39,78 & 56,49 & 59,76 & 63,72 & 64,96 & 1,60 & $<0,0001$ & 0,2412 & 0,3805 \\
\hline $\mathrm{MO}^{2}$ & 45,59 & 57,19 & 61,02 & 65,76 & 67,77 & 1,38 & $<0,0001$ & 0,5797 & 0,1400 \\
\hline $\mathrm{PB}^{3}$ & 28,08 & 64,88 & 65,55 & 63,55 & 62,81 & 2,79 & $<0,0001$ & $<0,0001$ & 0,0389 \\
\hline $\mathrm{EE}^{4}$ & 39,29 & 29,34 & 55,82 & 68,69 & 73,55 & 2,86 & $<0,0001$ & $<0,0001$ & $<0,0001$ \\
\hline $\mathrm{FDN}^{5}$ & 53,81 & 55,50 & 48,48 & 36,44 & 22,60 & 2,10 & $<0,0001$ & $<0,0001$ & 0,0773 \\
\hline $\mathrm{CT}^{6}$ & 47,55 & 55,44 & 59,07 & 64,21 & 67,33 & 1,33 & $<0,0001$ & $<0,0001$ & 0,0778 \\
\hline $\mathrm{CF}^{7}$ & 50,83 & 53,88 & 47,99 & 34,91 & 15,76 & 2,39 & $<0,0001$ & 0,0940 & 0,2687 \\
\hline $\mathrm{CNF}^{8}$ & 32,31 & 70,04 & 81,02 & 88,14 & 88,81 & 3,37 & $<0,0001$ & 0,0025 & 0,0021 \\
\hline
\end{tabular}

$\mathrm{EPM}=$ erro padrão da média; $\mathrm{L}=$ efeito linear; $\mathrm{Q}=$ efeito quadrático; $\mathrm{C}=$ efeito cúbico.

${ }^{1} \hat{\mathrm{Y}}=19,85+17,49 \mathrm{EM} \quad\left(\mathrm{R}^{2}=0.77\right) ; \quad{ }^{2} \hat{\mathrm{Y}}=27,20+15,20 \mathrm{EM} \quad\left(\mathrm{R}^{2}=0.79\right) ;{ }^{3} \hat{\mathrm{Y}}=-115,34+154,76 \mathrm{EM}-33,14 \mathrm{EM}^{2} \quad\left(\mathrm{R}^{2}=0.91\right)$; ${ }^{4} \hat{\mathrm{Y}}=129,19-123,13 \mathrm{EM}+38,90 \mathrm{EM}^{2} \quad\left(\mathrm{R}^{2}=0.72\right) ; \quad{ }^{5} \hat{\mathrm{Y}}=-34,47+117,27 \mathrm{EM}-35,86 \mathrm{EM}^{2} \quad\left(\mathrm{R}^{2}=0.91\right) ; \quad{ }^{6} \hat{\mathrm{Y}}=30,64+13,22 \mathrm{EM}$ $\left(\mathrm{R}^{2}=0.64\right) ;{ }^{7} \hat{\mathrm{Y}}=-61,31+147,06 \mathrm{EM}-44,07 \mathrm{EM}^{2}\left(\mathrm{R}^{2}=0.91\right) ;{ }^{8} \hat{\mathrm{Y}}=-12,82+40,03 \mathrm{EM}\left(\mathrm{R}^{2}=0,92\right)$.

Os coeficientes de digestibilidade da PB, da FDN e dos CF apresentaram comportamento quadrático ( $\mathrm{P}<0,001$; Tab. 4). Segundo Mertens (1992), a redução excessiva da quantidade de fibra nas dietas de ruminantes pode prejudicar a digestibilidade total dos alimentos, uma vez que, para manutenção das condições ótimas do rúmen, a fibra é fundamental, pois altera as proporções de ácidos graxos voláteis, em especial a relação acetato:propionato, estimula a mastigação e mantém o pH em níveis adequados à atividade microbiana.
Foi detectado efeito dos níveis de EM sobre os tempos de alimentação e ócio, expressos em $\mathrm{h} /$ dia, os quais foram influenciados de forma quadrática $(\mathrm{P}<0,05$; Tab. 5). O tempo máximo de alimentação e o tempo mínimo de ócio foram estimados em seis e nove horas, sete horas/dia, respectivamente, para os níveis de 1,61 e $1,58 \mathrm{Mcal} / \mathrm{kg}$ de MS. Quanto menor a ingestão de fibra, menor será o tempo despendido pelos animais em ingestão, aumentando, assim, o tempo em que estes permanecem em ócio (Van Soest, 1994).

Tabela 5. Tempo de alimentação (TAL), eficiência de alimentação (EAL), tempo de ruminação (TRU), eficiência de ruminação (ERU), tempo de mastigação total (TMT) e ócio, em função dos níveis de EM das rações experimentais, em carneiros da raça Somalis Brasileira

\begin{tabular}{|c|c|c|c|c|c|c|c|c|c|}
\hline \multirow{2}{*}{ Variáveis } & \multicolumn{5}{|c|}{ Níveis de EM (Mcal/kg de MS) } & \multirow{2}{*}{ EPM } & \multicolumn{3}{|c|}{ Efeito } \\
\hline & 1,18 & 2,07 & 2,25 & 2,42 & 2,69 & & $\mathrm{~L}$ & $\mathrm{Q}$ & $\mathrm{C}$ \\
\hline TAL (h/dia) ${ }^{1}$ & 5,48 & 6,33 & 4,52 & 3,90 & 3,67 & 0,22 & 0,0021 & 0,0049 & 0,0022 \\
\hline $\mathrm{EAL}(\mathrm{g} \mathrm{MS} / \mathrm{h})^{2}$ & 73,79 & 87,67 & 163,90 & 199,16 & 192,68 & 10,68 & $<0,0001$ & 0,1255 & 0,0100 \\
\hline EAL $(\mathrm{g} \mathrm{FDN} / \mathrm{h})^{3}$ & 60,25 & 60,61 & 80,53 & 71,12 & 47,54 & 3,51 & 0,8306 & 0,0331 & 0,0237 \\
\hline TRU (h/dia) ${ }^{4}$ & 8,41 & 8,61 & 6,77 & 7,84 & 7,20 & 0,28 & 0,1401 & 0,6836 & 0,4328 \\
\hline ERU $(\mathrm{g} \mathrm{MS} / \mathrm{h})^{5}$ & 47,48 & 64,06 & 130,77 & 100,31 & 90,50 & 8,36 & 0,0235 & 0,3775 & 0,0809 \\
\hline ERU $(\mathrm{g} \mathrm{FDN} / \mathrm{h})^{6}$ & 39,59 & 44,04 & 64,05 & 35,28 & 22,44 & 4,10 & 0,4103 & 0,0126 & 0,4212 \\
\hline TMT (h/dia $)^{7}$ & 13,89 & 14,95 & 11,29 & 11,75 & 10,87 & 0,43 & 0,0119 & 0,1074 & 0,0571 \\
\hline Ócio (h/dia) ${ }^{8}$ & 10,12 & 8,98 & 12,63 & 12,03 & 13,60 & 0,43 & 0,0058 & 0,0319 & 0,0956 \\
\hline
\end{tabular}

$\mathrm{EPM}=$ erro padrão da média; $\mathrm{L}=$ efeito linear; $\mathrm{Q}=$ efeito quadrático; $\mathrm{C}=$ efeito cúbico.

${ }^{1} \hat{Y}=-0,15+7,62 \mathrm{EM}-2,36 \mathrm{EM}^{2}\left(\mathrm{R}^{2}=0.77\right) ;{ }^{2} \hat{\mathrm{Y}}=-46,00+89,10 \mathrm{EM}\left(\mathrm{R}^{2}=0.88\right) ;{ }^{3} \hat{\mathrm{Y}}=-40,49+123,20 \mathrm{EM}-32,93 \mathrm{EM}^{2}\left(\mathrm{R}^{2}=0.83\right) ;$ ${ }^{4} \tilde{\mathrm{Y}}=7,77 ;{ }^{5} \hat{\mathrm{Y}}=6,98+37,58 \mathrm{EM}\left(\mathrm{R}^{2}=0.74\right) ;{ }^{6} \hat{\mathrm{Y}}=-88,74+160,33 \mathrm{EM}-44,14 \mathrm{EM}^{2}\left(\mathrm{R}^{2}=0.74\right) ;{ }^{7} \hat{\mathrm{Y}}=17,12-2,14 \mathrm{EM}\left(\mathrm{R}^{2}=0.68\right) ;$ ${ }^{8} \hat{\mathrm{Y}}=18,48-11,47 \mathrm{EM}+3,63 \mathrm{EM}^{2}\left(\mathrm{R}^{2}=0.71\right)$. 
As eficiências de alimentação e ruminação, em $g$ FDN/h, apresentaram efeito quadrático $(\mathrm{P}<0,05$; Tab. 5). Para as eficiências de alimentação e de ruminação, em g MS/h, houve efeito linear crescente $(\mathrm{P}<0,05)$ à medida que os níveis de EM das rações aumentaram. Segundo Van Soest (1994), o tempo despendido em ruminação é influenciado pela natureza da dieta, sendo proporcional ao teor de parede celular dos volumosos. Desta forma, em dietas com proporções maiores de concentrado será observada maior eficiência de ruminação ou mastigação. As inferências de Dado e Allen (1995) reiteram as observações de Van Soest, ao afirmarem que o número de períodos de ruminação aumenta de acordo com o teor de fibra da dieta, havendo a necessidade de processamento da digesta ruminal para elevar a eficiência digestiva.

O tempo de mastigação total apresentou efeito linear decrescente $(\mathrm{P}<0,05$; Tab. 5), uma vez que, quando decrescem os constituintes da parede celular da dieta, decresce o tempo total de mastigação (Dulphy et al., 1980). Resultados semelhantes foram observados por Fontenele $e t$ al. (2011) e Camilo et al. (2012). O número de bolos ruminais, o de mastigações merícicas e de mastigações merícicas por bolo ruminal e o tempo de mastigações merícicas por bolo ruminal não foram influenciados $(\mathrm{P}>0,05)$ pelos níveis de energia metabolizável nas rações (Tab. 6).

Tabela 6. Número de bolos ruminais (NBR), número de mastigações merícicas por dia (MMnd), número mastigações merícicas por bolo ruminal (MMnb) e tempo de mastigações merícicas por bolo ruminal (MMtb), em função dos níveis de EM das rações experimentais, em carneiro da raça Somalis Brasileira

\begin{tabular}{|c|c|c|c|c|c|c|c|c|c|}
\hline \multirow{2}{*}{ Variáveis } & \multicolumn{5}{|c|}{ Níveis de EM (Mcal/kg de MS) } & \multirow{2}{*}{ EPM } & \multicolumn{3}{|c|}{ Efeito } \\
\hline & 1,18 & 2,07 & 2,25 & 2,42 & 2,69 & & $\mathrm{~L}$ & $\mathrm{Q}$ & $\mathrm{C}$ \\
\hline $\mathrm{NBR}\left(\mathrm{n}^{\circ} / \mathrm{dia}\right)^{1}$ & 706,88 & 675,01 & 528,28 & 624,43 & 608,57 & 24,70 & 0,1254 & 0,6407 & 0,4081 \\
\hline MMnd(n $\%$ dia $)^{2}$ & 43970,46 & 46180,44 & 33747,66 & 43340,84 & 36565,81 & $1.740,80$ & 0,2024 & 0,5698 & 0,6460 \\
\hline $\operatorname{MMnb}\left(\mathrm{n}^{\circ} / \mathrm{bol}\right)^{3}$ & 61,85 & 68,25 & 66,40 & 70,08 & 61,24 & 1,55 & 0,6203 & 0,0744 & 0,4112 \\
\hline $\operatorname{MMtb}(\mathrm{seg} / \mathrm{bol})^{4}$ & 42,78 & 46,28 & 48,68 & 46,06 & 43,70 & 1,11 & 0,5214 & 0,1300 & 0,6317 \\
\hline
\end{tabular}

$\mathrm{EPM}=$ erro padrão da média; $\mathrm{L}=$ efeito linear; $\mathrm{Q}=$ efeito quadrático; $\mathrm{C}=$ efeito cúbico.

${ }^{1} \tilde{Y}=628,63 ;{ }^{2} \tilde{Y}=40761.04 ;{ }^{3} \tilde{Y}=65,56 ;{ }^{4} \tilde{Y}=45,50$.

Em relação ao consumo, a soma dos períodos $1 \mathrm{e}$ 2 , referentes aos intervalos das seis às $12 \mathrm{~h}$ e das 12 às $18 \mathrm{~h}$, correspondeu ao maior tempo despendido para o consumo $(69,64 \%$ do consumo total), mostrando que esta atividade concentrou-se durante o dia (Fig. 1). Essas observações foram registradas também por Pereira et al. (2009) e Macedo et al. (2007), que obtiveram médias de $83,92 \%$ e $57,12 \%$, respectivamente, do tempo gasto com alimentação somando os períodos 1 e 2 . Segundo Dado e Allen (1995), a ingestão de MS é elevada após o fornecimento da ração, quando o alimento ainda está fresco. Sendo os ruminantes animais de hábito diurno, a atividade de alimentação é mais frequente durante o dia que à noite, contudo esse comportamento pode variar (Forbes, 1995).
Em relação à atividade de ruminação (Fig. 2), esta ocorreu principalmente à noite $(55,29 \%)$, entre os períodos 3 e 4 (das 18 às 24 h e das 24 às seis horas), respectivamente. $\mathrm{O}$ padrão diário da atividade de ruminação iniciou-se 10 horas após o fornecimento da alimentação (período 3) e atingiu os valores máximos durante as seis horas subsequentes (período 4). Polli et al. (1996) registraram que a atividade de ruminação é influenciada pela atividade de alimentação e ocorre após os períodos de ingestão do alimento, com o animal em estado de repouso. Segundo Fischer et al. (1998), os animais ruminam preferencialmente à noite, porém são também ritmados pelo fornecimento de alimento e sofrem forte influência da relação volumoso:concentrado da dieta. 


\section{Aquino et al.}

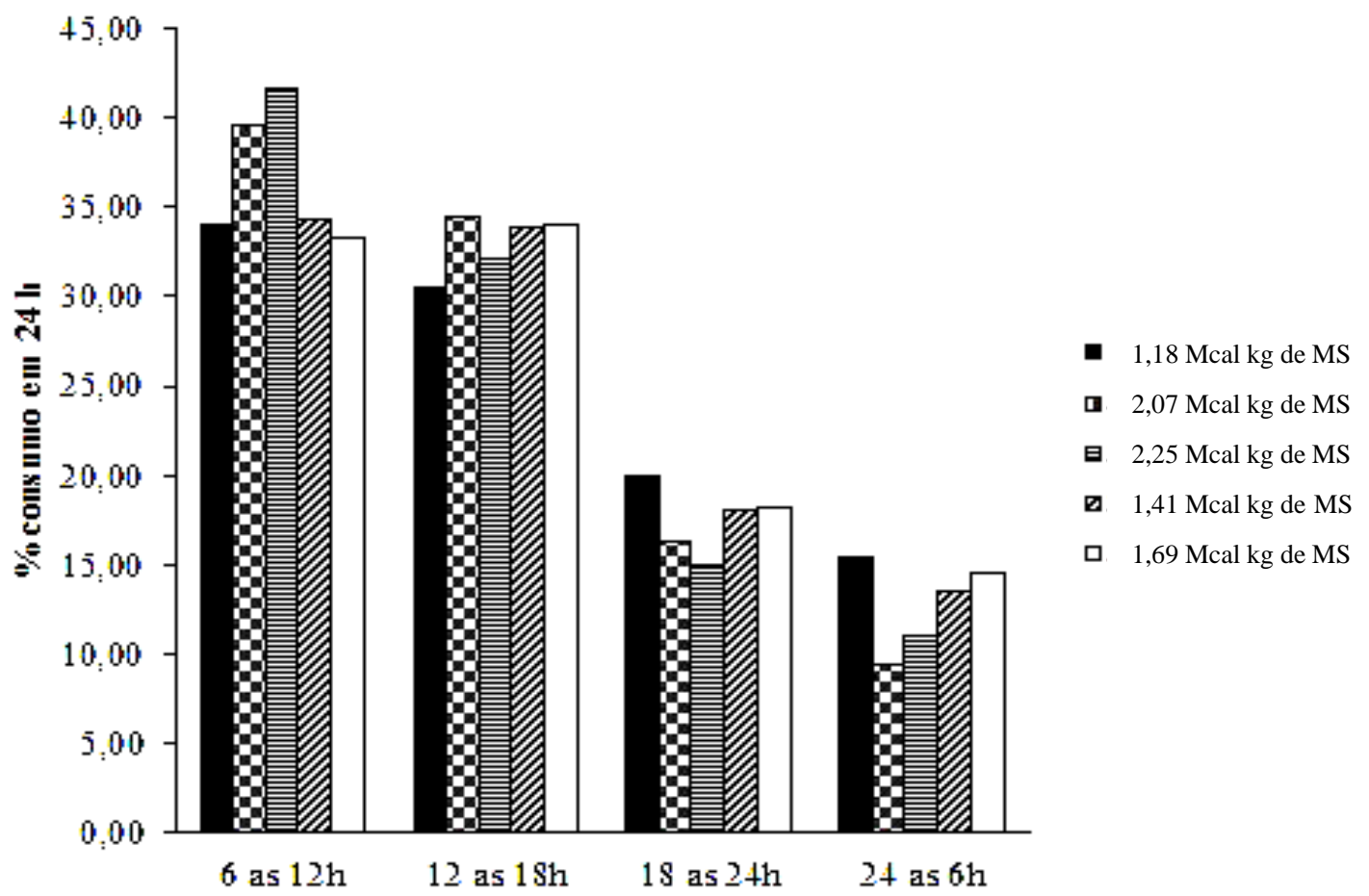

Figura 1. Distribuição das atividades de consumo em quatro períodos de tempo (das seis às $12 \mathrm{~h}$; 12 às $18 \mathrm{~h} ; 18$ às $24 \mathrm{~h}$ e das 24 às seis horas) do dia, em carneiros da raça Somalis Brasileira.

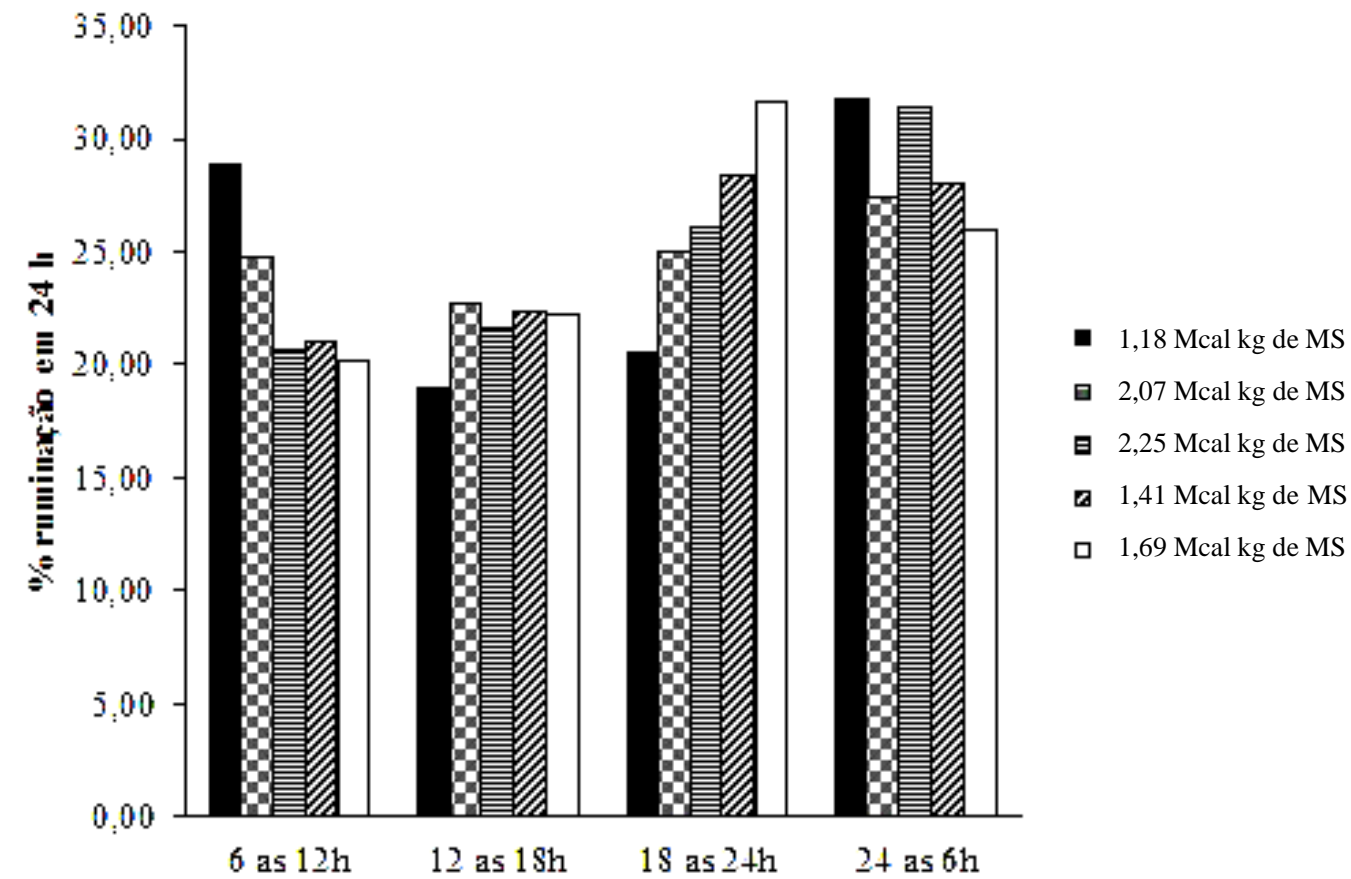

Figura 2. Distribuição das atividades de ruminação em quatro períodos de tempo (das seis às $12 \mathrm{~h} ; 12$ às $18 \mathrm{~h} ; 18$ às $24 \mathrm{~h}$ e das 24 às seis horas) do dia, em carneiros da raça Somalis Brasileira. 


\section{CONCLUSÃO}

O aumento dos níveis de energia metabolizável das rações influencia o consumo, a digestibilidade dos nutrientes e o comportamento ingestivo de cordeiros Somalis Brasileira.

\section{AGRADECIMENTOS}

Os autores agradecem ao Conselho Nacional de Pesquisa e Desenvolvimento Científico e Tecnológico (CNPq) pelo suporte financeiro.

\section{REFERÊNCIAS}

CAMILO, D.A.; PEREIRA, E.S.; PIMENTEL, P.G. et al. Intake and feeding behaviour of Morada Nova lambs fed different energy levels. Ital. J. Anim. Sci., v.11, p.13-19, 2012.

CASALI, A.O.; DETMANN, E.; VALADARES FILHO, S.C. et al. Estimação de teores de componentes fibrosos em alimentos para ruminantes em sacos de diferentes tecidos. Rev. Bras. Zootec., v.38, p.130-138, 2009.

DADO, R.G.; ALLEN, M.S. Intake limitations, feeding behavior, and rumen function of cows challenged with rumen fill from dietary or inert bulk. J. Dairy Sci., v.78, p.119-133, 1995.

DULPHY, J.P.; REMOND, B.; THERIEZ, M. Ingestive behavior and related activities in ruminants. In: RUCKEBUSH, Y.; THIVEND, P. (Eds.) Digestive physiology and metabolism in ruminants. Lancaster: MTP, 1980. p.103-122.

FISCHER, V.; DESWYSEN, A.G.; DÈPRES, L. et al. Padrões nectemerais do comportamento ingestivo de ovinos. Rev. Bras. Zootec., v.27, p.362-369, 1998.

FONTENELE, R.M.; PEREIRA, E.S.; CARNEIRO, M.S.S. et al. Consumo de nutrientes e comportamento ingestivo de cordeiros da raça Santa Inês alimentados com rações com diferentes níveis de energia metabolizável. Rev. Bras. Zootec., v.40, p.12801286, 2011.

FORBES, J.M. Voluntary food intake and diet selection in farm animals. Wallington: $\mathrm{CAB}$ International, 1995. 532p.
HALL, M.B. Calculation of non-structural carbohydrate content of feeds that contain nonprotein nitrogen. Gainesville: University of Florida, 2000. p.25 (Bulletin 339).

JOHNSON, T.R.; COMBS, D.K. Effects of prepartum diet, inert rumen bulk, and dietary polythylene glicol on dry matter intake of lactating dairy cows. J. Dairy Sci., v.74, p.933944, 1991.

MACEDO, C.A.B.; MIZUBUTI, I.Y.; MOREIRA, F.B. et al. Comportamento ingestivo de ovinos recebendo dietas com diferentes níveis de bagaço de laranja em substituição à silagem de sorgo na ração. Rev. Bras. Zootec., v.36, p.1910-1916, 2007.

MERTENS, D.R. Análise da fibra e sua utilização na avaliação e formulação de rações. In: SIMPÓSIO INTERNACIONAL DE RUMINANTES, REUNIÃO ANUAL DA SOCIEDADE BRASILEIRA DE ZOOTECNIA, 29., 1992, Lavras. Anais... Lavras: Sociedade Brasileira de Zootecnia, 1992. p.188-219.

MERTENS, D.R. Regulation of forage intake. In: FAHEY JUNIOR, G.C. (Ed.) Forage quality, evaluation and utilization. Madison: American Society of Agronomy, 1994. p.450-493.

NUTRIENT requirements of beef cattle. 8.ed. Washington: National Academy Press, 2000. 248p.

NUTRIENT requeriments of dairy cattle. Washington: National Academy Press, 2001. $57 \mathrm{p}$.

NUTRIENT requirements of sheep. Washington: D.C.: National Academic Press, 2007. 362p.

OFFICIAL methods of analysis. 15.ed. Washington: AOAC. 1990. 1117p.

PEREIRA, E.S.; MIZUBUTI, I.Y.; RIBEIRO, E.L.A. et al. Consumo, digestibilidade aparente dos nutrientes e comportamento ingestivo de bovinos da raça Holandesa alimentados com dietas contendo feno de capim-tifton 85 com diversos tamanhos de partícula. Rev. Bras. Zootec., v.38, p.190-195, 2009.

POLLI, V.A.; RESTLE, J.; SENNA, D.B. et al. Aspectos relativos à ruminação de bovinos $\mathrm{e}$ bubalinos em regime de confinamento. Rev. Bras. Zootec., v.25, p.987-993, 1996. 
RUSSELL, J.B.; O'CONNOR, J.D.; FOX, D.G. et al. A net carbohydrate and protein system for evaluating cattle diets. 1 . Rumen fermentation. $J$. Anim. Sci., v.70, p.3551-3561, 1992.

SILVA, R.R.; MAGALHÃES, A.F.; CARVALHO, G.G.P. et al. Comportamento ingestivo de novilhas mestiças de holandês suplementadas em pastejo de brachiaria decumbes. Aspectos metodológicos. Arch. Zootec., v.5, p.1-7, 2004.

SNIFFEN, C.J.; O'CONNOR, J.D.; VAN SOEST, P.J. et al. A net carbohydrate and protein system for evaluating cattle diets: II. Carbohydrate and protein availability. J. Dairy Sci., v.70, p.3562-3577, 1992.

STATISTICAL system for windows - SAS. Release 9.1. Cary, NC: SAS Institute, 2003.
VALADARES FILHO, S.C. Digestão total $e$ parcial da matéria seca e carboidratos em bovinos e bubalinos. 1985. 148f. Tese (Doutorado em Zootecnia) - Universidade Federal de Viçosa, Viçosa.

VAN SOEST, P.J.; ROBERTSON, J.B.; LEWIS, B.A. Methods for dietary fiber, neutral detergent fiber and nonstarch polysaccharides in relation to animal nutrition. J. Dairy Sci., v.74, p.35833597, 1991.

VAN SOEST, P.J. Nutritional ecology of the ruminant. 2.ed. London: Comstock Publishing Associates, 1994. 476p.

WEISS, W.P. Energy prediction equations for ruminant feeds. In: CORNELL NUTRITION CONFERENCE FOR FEED MANUFACTURERS, 61., 1999, Ithaca. Proceedings... Ithaca: Cornell University, 1999. p.176-185. 